\title{
Control System for a Diesel Generator and UPS Based Microgrid
}

\author{
Andriy Palamar, Tallinn University of Technology, Elmo Pettai, Tallinn University of Technology, \\ Viktor Beldjajev, Tallinn University of Technology
}

\begin{abstract}
In this paper a microgrid composed of a diesel generator and two uninterruptible power supply systems with separate battery banks is introduced. The microgrid located in three academic buildings of Tallinn University of Technology. A three-level control and monitoring system for the microgrid based on the EtherNet/IP communication network is developed. In addition, a control strategy of the microgrid in the gridconnected and stand-alone mode of operation is proposed.
\end{abstract}

Keywords: control system, diesel generator, microgrid, UPS

\section{INTRODUCTION}

In recent years demand in the continuity of power supply in the local distributed areas is steadily increasing. Nowadays, more and more consumers (e.g. data centers, information laboratories, computer servers) require constant availability of electrical energy. In such cases the installation of uninterruptible power supply (UPS) units with energy storage devices are necessary. Therewith, to achieve opportunities of longer interruptions it needs a large capacity of battery systems which increase the equipment cost. To overcome this problem, UPS units with distributed generation are commonly used. Distributed generation encompasses a wide range of prime technologies, such as internal combustion engines, gas turbines, microturbines, photovoltaic, fuel cells, and windpower [1]. Despite the fact that renewable energy resources are well suited for electricity generation, their application involves a major problem of unstable energy provision due to the fluctuating nature of resources [2]. In addition, they have relatively low efficiency and high cost.

On the other hand, a diesel powered generator has been extensively used as emergency and standby power, to meet peak loads and for electricity supply in isolated power systems [3]. It still remains popular for a number of compelling reasons, such as: low cost, availability, efficiency, reliability, flexibility, safety [4]. A better way to realize the emerging potential of distributed generation, e.g. a diesel generator, is to take a system approach which views generation and associated loads as a microgrid [5].

Microgrid is a concept of defining the operation of distributed generation, in which different microsources operate as a single controllable system that provides power and heat to a cluster of loads in the local area [6] - [8]. The most positive features of microgrids are the (i) relatively short distances between generation and loads, (ii) low generation and distribution voltage level. The primary goal of microgrid architectures is to significantly improve energy production and delivery to load customers, while facilitating a more stable electrical infrastructure [9]. Consequently, a combination of a diesel generator and UPS in a microgrid system is a solution to guarantee a continuity of power supply with relatively small expenditures on equipment.

Despite many advantages of the microgrid there remain many technical challenges and difficulties. One of them is the design, acceptance, and availability of low-cost technologies for installing and using microgrids [10]. The increased deployment of power electronic devices in distributed energy sources within microgrids requires effective monitoring and control systems for safe and stable operation [11]. Realization of complicated controlling processes in microgrids requires a specific communication infrastructure and protocols. It is required to organize free access to the network and efficient allocation of costs.

This paper proposes a microgrid system to be located in three academic buildings of Tallinn University of Technology (TUT). This microgrid includes a diesel generator and two UPS systems, each with separate battery storage. The main goal of this work is to develop an intelligent control system of the microgrid that is efficient enough to manage itself for power balance by making use of state of the art communication technology. In addition, the aim of this paper is to describe the control strategy of the microgrid in both grid-connected and stand-alone mode of operation.

The proposed micro-grid system is capable of upgrading the power grid of TUT by making it more reliable, secure, efficient, and de-centralized, by providing uninterruptible power supply functions, and minimizing emissions and system losses. The control system of this microgrid will enhance the reliability and stability of the power system on the one hand and will make the microgrid an easy to use product on the other hand.

\section{GENERAL OVERVIEW OF A MICROGRID}

A microgrid is described as a small (several MW or less in scale) power system with three primary components: distributed generators with optional storage devices, autonomous load centers, and system capability to operate interconnected with or islanded from the larger utility electrical grid [9], [12] - [13].

Multiple facility microgrids span multiple buildings or structures, with loads typically ranging between $2 \mathrm{MW}$ and $5 \mathrm{MW}$, such as campuses (medical, academic, municipal, etc), military bases, industrial and commercial complexes, and building residential developments [14]. 


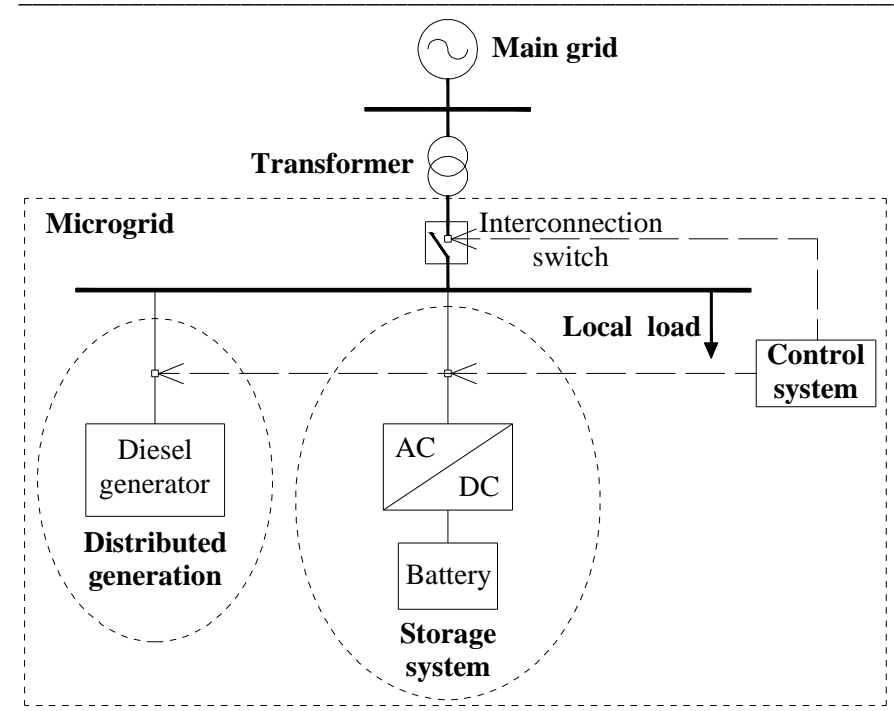

Fig. 1. Schematic diagram of a microgrid.

A schematic diagram of a microgrid is shown in Fig. 1. A microgrid can be a DC or an AC grid. An AC microgrid can be a single-phase or a three-phase system. It can be connected to low voltage or medium voltage power distribution networks [15]. This paper considers only an AC microgrid that is connected to a city power grid.

Generally, a microgrid includes four basic technologies for operation [6], [10]: distributed generation units, distributed storage devices, an interconnection switch, and a control system.

\section{A. Distributed Generation Units}

Distributed generation units are small sources of energy located at or near the point of use. There are two basic classes of microsources; one is a DC source (fuel cells, photovoltaic cells, etc.), the other is a high frequency AC source (microturbines, reciprocating engine generators, wind generators) that needs to be rectified.

\section{B. Distributed Storage Devices}

Distributed storage devices are used in microgrid applications where the generation and loads of the microgrid cannot be exactly matched. There are several forms of energy storage, such as the batteries, supercapacitors, and flywheels. Distributed storage enhances microgrid systems overall performance in three ways. First, it stabilizes and permits distributed generation units to run at a constant and stable output, despite load fluctuations. Second, it provides the ride through capability when there are dynamic variations of primary energy (such as those of sun, wind, and hydropower sources). Third, it permits distributed generation to seamlessly operate as a dispatchable unit. Moreover, energy storage can benefit power systems by damping peak surges in electricity demand, countering momentary power disturbances, providing outage ride through while backup generators respond, and reserving energy for future demand.

\section{Interconnection Switch}

The interconnection switch is the point of connection between the microgrid and the rest of the distribution system. New technologies in this area consolidate the various power and switching functions (power switching, protective relaying, metering, and communications) traditionally provided by relays, hardware, and other components at the utility interface into a single system with a digital signal processor. The interconnection switches are designed to meet grid interconnection standards.

\section{Control System}

A microgrid may operate either connected to the main grid or disconnected from it. Hence, there are two steady states of operation, grid-connected and islanded. Moreover, there are two transient modes of operation of the microgrid: transfer from grid-connected mode to islanded mode and vice versa [16]. The control system of a microgrid is designed to safely operate the system in both of these modes. This system may be based on a central controller or embedded as autonomous parts of each distributed generator. When the utility is disconnected, the control system must control the local voltage and frequency, provide (or absorb) the instantaneous real power difference between generation and loads, provide the difference between generated reactive power and the actual reactive power consumed by the load, and protect the internal microgrid.

\section{STRUCTURE OF THE PROPOSED MiCROGRID}

The microgrid is to be located in three buildings of TUT: Faculty of Power Engineering, TUT Library, School of Economics and Business Administration. Consequently, according to the classification given in [14], this power system can be defined as a multiple facility microgrid. Fig. 2 illustrates the various components of the power system of the microgrid at TUT.

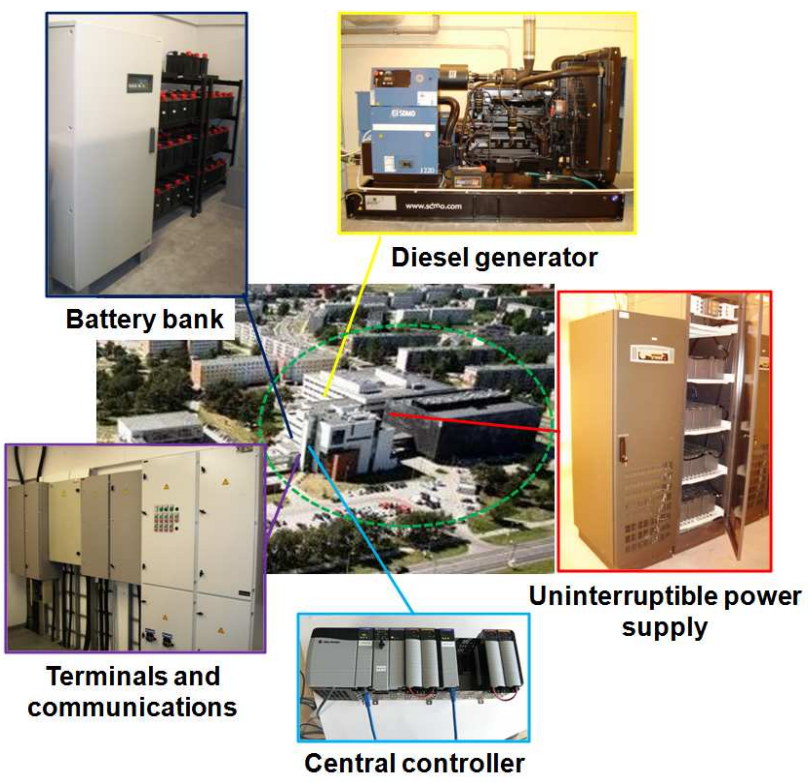

Fig. 2. Microgrid at Tallinn University of Technology. 


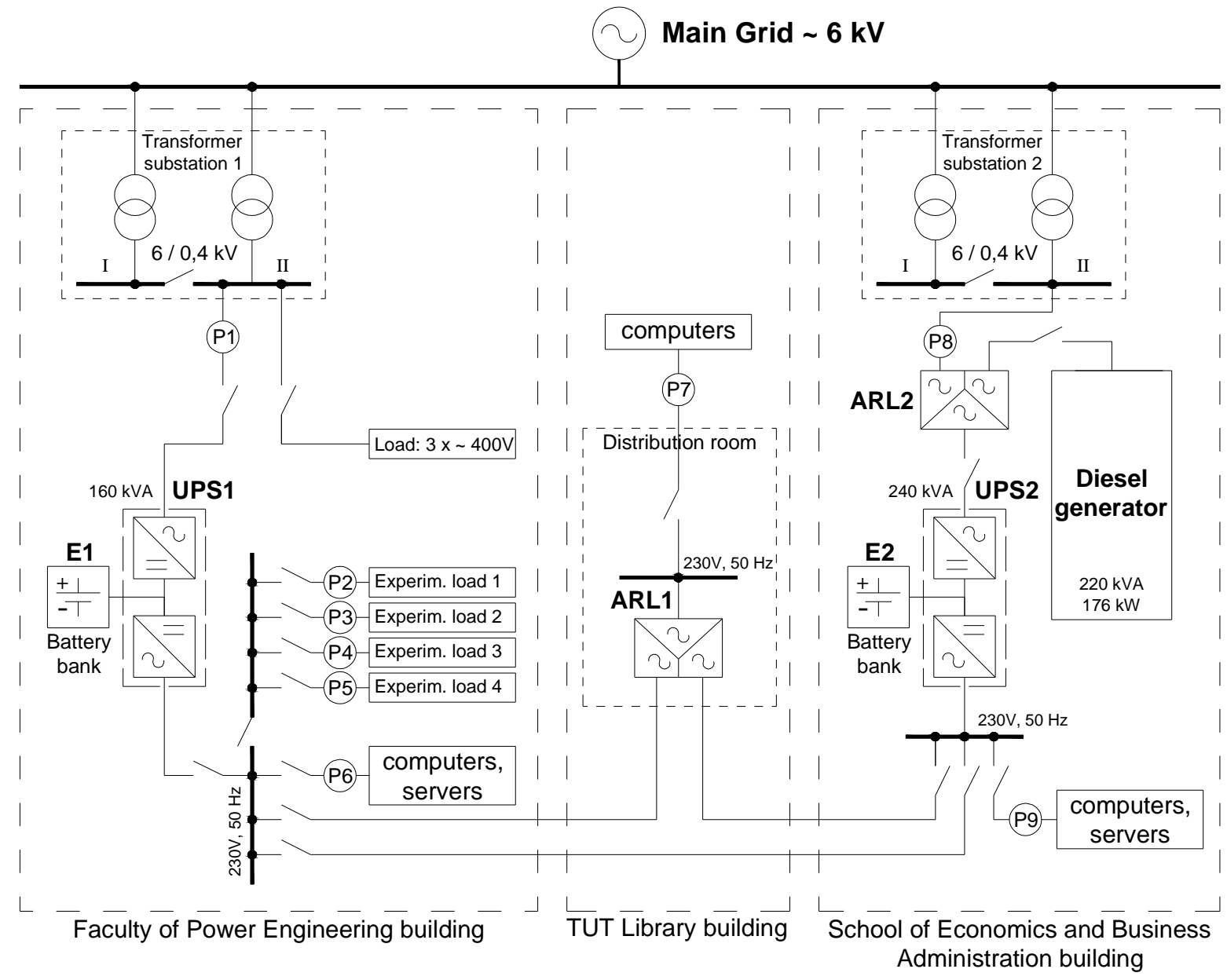

Fig. 3. Structural scheme of the microgrid for Tallinn University of Technology.

The structure of the microgrid for TUT campuses is shown in Fig. 3. Microgrid system targeted in this study is autonomous areas with the power demand of several kilowatts including a diesel generator, two UPS units with battery storages, and loads. They are connected to the power network via power electronic switches forming the local three-phase $400 \mathrm{~V} \mathrm{AC} \mathrm{microgrid} \mathrm{with} \mathrm{a} \mathrm{frequency} \mathrm{of} 50 \mathrm{~Hz}$.

The diesel generator is used as the main distributed energy resource in this microgrid. It has a nominal power of $176 \mathrm{~kW} / 220 \mathrm{kVA}$, a voltage of $230 \mathrm{~V} / 400 \mathrm{~V}$ and a maximum current of $318 \mathrm{~A}$. This generator is connected to the AC network via the automatic relay logic (ARL2). The ARL2 is continuously observing both sides: the main grid and the microgrid. If there is a fault in the general grid, the ARL2 will disconnect the microgrid, creating this way an independent energetic island. Consequently, ARL2 is used as an interconnection switch for this microgrid.

The battery banks (E1 and E2) are used as the distributed energy storage devices in the microgrid to ensure continuous supply of the local load. They are connected to the electrical network through the two UPS systems: UPS1 (160 kVA) and UPS2 $(240 \mathrm{kVA})$. Hence, we can conclude that the microgrid has two main possible operation modes: grid-connected and islanded mode.
Main consumers of the microgrid are the computers and servers located in the laboratories and office rooms in three TUT buildings. The clients in the Library building (computers) are connected to the electrical network using ARL1. In addition, four experimental non-critical loads may be connected to the power system. Nine intelligent sensors (P1..P9) monitor the consumption of electrical energy by all these loads. Their task is to measure electrical parameters of the network, such as voltage, current, power, energy, power factor and transmit this information to a central controller.

The microgrid is connected to the general city power grid using two two-section transformer substations (6000 V / 400 V) located in the Faculty of Power Engineering and the School of Economics and Business Administration buildings.

\section{DESCRIPTION OF THE CONTROL SYSTEM}

Taking advantage of multiagent technologies [17], [18] the control system for the microgrid of TUT is developed with the following specifications:

- balance of electric demand and supply of power network are provided;

- both the steady state mode of operation and the transient sequences of the microgrid are achieved. 


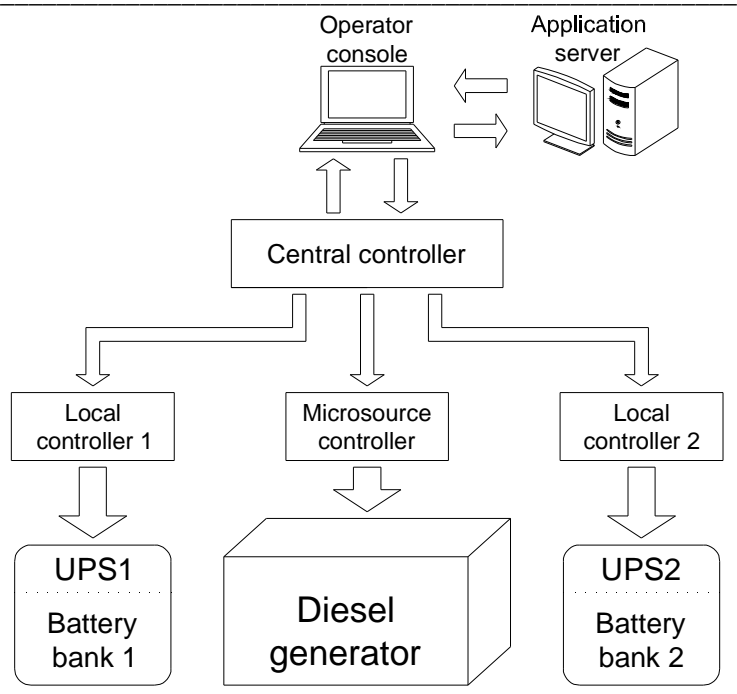

Fig. 4. Block diagram of the hierarchical control system of the microgrid.

A block diagram of the hierarchical control system is demonstrated in Fig. 4. The control structure of the microgrid has three levels:

1) operator console with an application server;

2) central controller (CC);

3) local controllers (LC).

\section{A. Operator Console}

The operator console heads the hierarchical control system. It is a computerized workstation with special software. Its main goals are: to keep track of the whole system by monitoring the status of the communication nodes, switches and microgrid components; to collect data from the measuring devices; to calculate supply and demand of power; to visualize information received; to display the basic modes of the microgrid; and to transfer control commands to the central controller. The function block diagram of the software is shown in Fig. 5. It comprises a supply and demand calculation unit, a monitoring and display unit, a control scheme, and a dispatching unit. An application server is designed for archiving data received from the measuring devices.

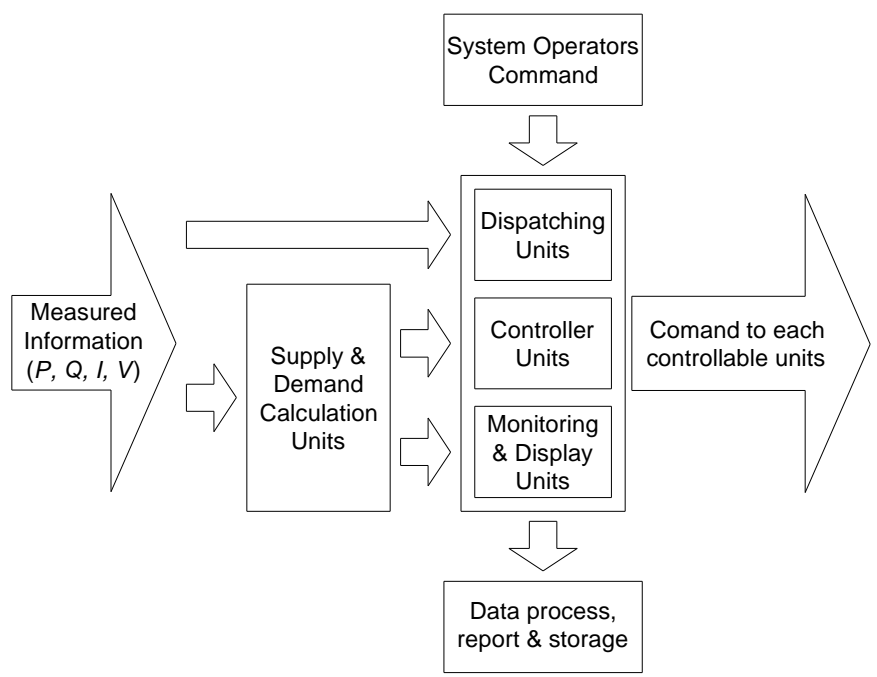

Fig. 5. Functional blocks of software units of the control system.

\section{B. Central Controller}

The central controller provides the main interface between the operator console and others communication nodes of the microgrid control system. It manages all the operations of the microgrid. The central controller operates in real time. Its main functions: collecting information from the measuring devices, transferring data from the operator console and the application server, managing the power supply switches, and transmitting the control commands to the local controllers.

\section{Local Controllers}

The group of the local controllers is related to the third hierarchical control level. They include a microsource controller that is located at the diesel generator. It manages active and reactive power production levels of the microsource. Moreover, the microsource controller is responsible for maintaining the desired steady-state and dynamic performance of the microgrid in the islanded mode. The other local controllers are located in the two UPS systems. Their main goals are to provide management of charge of the batteries storage.

\section{Measuring Process}

The measured data required for the proposed monitoring and control system are voltage, current, power, energy, and power factor. Real-time information is collected through the intelligent measuring devices located at the output of the energy source, at the input of each load, and at both of the UPS systems. In this system, Allen-Bradley Powermonitor 3000 [19] is used to measure these instantaneous values. Such operating information is displayed in real-time for monitoring and energy management purposes.

\section{E. Communication Network}

A communication infrastructure is necessary between the components of the control system (operator console, application server, central controller, and local controllers). It has to provide three services: control, configuration, and collection of data. The availability of communication infrastructure introduces automation of the management process with minimum human influence.

The Ethernet/IP industrial protocol has been chosen in this microgrid as a communication network for data transfer for all those control units. Ethernet is quickly becoming the effective local area network (LAN) for electronic communication. It now delivers highly reliable, low-cost 10/100/1000 Mbps performance over long distances [20]. The adoption of the standard protocol allows designing and developing modular solutions using off-the-shelf, low-cost, widely available, and fully supported hardware and software components. The main advantages of using Ethernet/IP protocol are: the transition from a centralized control to a distributed control; wiring reduction - no need for point to point connections. This solution provides flexibility and scalability for low-cost implementations. 


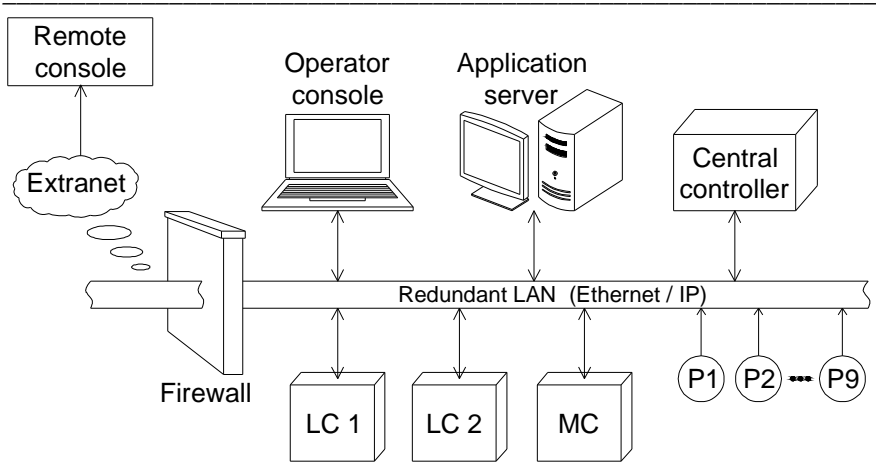

Fig. 6. Block diagram of the microgrid communication network of the control system.

The block diagram of the microgrid communication network is illustrated in Fig. 6. Every communication node has to get a registered IP address to the application server. The amount of data to be exchanged between network control units includes mainly commands to the central controller and the local controllers, measured data from intelligent sensors to the $\mathrm{CC}$ and operator console, and messages to control microgrid switches. Furthermore, this topology provides an opportunity for immediate control center access via remote consoles and web based laptops for necessary actions to be taken.

\section{V.MANAGEMENT OF THE MICROGRID}

Tasks of the control system depend on the mode in which the microgrid operates.

\section{A. Grid-Connected Mode}

In this operation mode, the microgrid is connected to the main grid through the two transformer substations. The main grid produces power to the local loads of TUT. Hence, the balance between the generation and consumption as well as the control of the electrical parameters of the system is set by the city grid.

The aim of the UPS1 and UPS2 is to obtain energy backup as much as possible. Consequently, during grid-connected operation, the microgrid must charge the battery banks E1 and E2. Concerning the central controller, the purpose is to optimize the microgrid performance.

\section{B. Transition from Grid-Connected to Islanded Mode}

The instant at which the islanding occurs must be detected immediately in order to guarantee a continuity of power supply to the microgrid. There are various islanding detection methods proposed for microgrid systems [21]. In this case study, the detection is achieved using an algorithm described in [22].

When the fault of the main grid occurs, the central controller disconnects the microgrid from the main grid, and keeps the uninterrupted power supply of the important loads. For this purpose, the intelligent sensors P1 and P8 have to detect that the voltage is below the normal level and the central controller must send the command to open the interconnection switches.

\section{Islanded Mode}

When the islanded operation mode is detected, the ARL2 disconnects the electrical network of the microgrid from the main grid and connects it to the diesel generator. Then diesel generator will start to produce electrical power. During the time it takes to detect the outage, disconnect the main grid, and start the diesel generator, the sensitive loads are supplied from the battery bank E2 with UPS2. When the diesel generator has reached normal operation mode, the UPS2 starts to charge the battery.

In the islanded mode, two requirements have to be fulfilled: the power balance between the generation and the consumption and the control of the primary electrical parameters of the microgrid, such as voltage and frequency.

Moreover, the battery storage E1 with UPS1 is responsible for ensuring the power balance of the microgrid by means of absorbing and injecting the power deviation between the diesel generator and the local consumers. If all the available power provided by the diesel generator is not sufficient to feed the local loads, the control system will detach non-critical loads (experimental loads 1..4).

The central controller is responsible for monitoring the operation of the microgrid and coordinating the local controllers. The control system provides voltage control to ensure all consumers voltages are within their normal ranges. The main aim of the control system in the islanded mode is to increase the microgrid reliability by guaranteeing local load supply.

\section{Transition from Islanded to Grid-Connected Mode}

When the microgrid is working in the islanded mode and the measuring devices P1 and P8 have detected that the voltage in the main grid is stable and fault-free, the central controller has to resynchronize the microgrid to the frequency, amplitude and phase of the grid, in order to reconnect seamlessly the microgrid. Once the voltage in the microgrid is synchronized with the utility voltage, the microgrid can be reconnected to the grid and the central controller will pass from a voltage control mode to a power control mode. In the same way if non-critical loads are detached, they are also reconnected. After that, switches will close, interconnecting the microgrid to the main grid. After the grid restoration, diesel generator stops to produce the power.

\section{CONCLUSIONS}

In this paper the architecture of the microgrid for three academic buildings of Tallinn University of Technology has been introduced. This microgrid includes a diesel generator and two UPS systems with battery storages. The three-level control and monitoring system for the microgrid based on the EtherNet/IP communication network was developed. Furthermore, the control strategy of the microgrid in the gridconnected and islanded mode was proposed. This control system will enable the microgrid system to balance the electric power demand and supply and to simultaneously control the state of the power network of TUT. 


\section{ACKNOWLEDGEMENT}

This paper was supported by the Project DAR8130 "Doctoral School of Energy and Geotechnology II".

\section{REFERENCES}

1] A. P. Agalgaonkar, S. V. Kulkarni, S. A. Khaparde, and S. A. Soman, "Placement and Penetration of Distributed Generation Under Standard Market Design," International Journal of Emerging Electric Power Systems, vol. 1, no. 1, pp. 1-18, 2004

[2] Z. Bilic, H. Glavas, and D. Sljivac, "Designing the Optimal Stand alone Power System which uses Wind Power and Solar Radiation for Remote Area Object," in International Youth Conference on Energetic, Leverkusen, Germany, 2007.

[3] D. Canever, G. J. W Dudgeon, S. Massucco, J. R. McDonald, and F. Silvestro, "Model Validation and Coordinated Operation of a Photovoltaic Array and a Diesel Power Plant for Distributed Generation," in Power Engineering Society Summer Meeting, vol. 1, Vancouver, Canada, 2001, pp. 626-631.

[4] J. W. Herzog, "Current and Near-Term Emission Control Strategies for Diesel Powered Generator Sets," in Telecommunications Energy Conference, Montreal, Canada, 2002, pp. 394-399.

[5] R. Lasseter, A. Akhil, C. Marnay, J. Stephens, J. Dagle, R. Guttromson, A. S. Meliopoulous, R. Yinger, and J. Eto, "White Paper on Integration of Distributed Energy Resources. The CERTS Microgrid Concept," Consortium for Electric Reliability Technology Solutions, CA, Tech. Rep. LBNL-50829, 2002.

[6] R. Lasseter, "Microgrids," in Power Engineering Society Winter Meeting, vol. 1, New York, NY, 2002, pp. 305-308.

[7] A. Mohamed, "Microgrid Modeling and Online Management," $\mathrm{PhD}$ thesis, Helsinki University of Technology, Helsinki, Finland, 2008.

8] D. Yubing, G. Yulei, L. Qingmin, and W. Hui, "Modelling and Simulation of the Microsources Within a Microgrid," in Electrical Machines and Systems, Jinan, China, 2008, pp. 2667-2671.

[9] C. M. Colson and M. H. Nehrir, "A Review of Challenges to Real-Time Power Management of Microgrids," in IEEE Power \& Energy Society General Meeting, Calgary, Canada, 2009, pp. 1-8.

[10] B. Kroposki, T. Basso, and R. DeBlasio, "Microgrid Standards and Technologies," in Power and Energy Society General Meeting Conversion and Delivery of Electrical Energy in the 21st Century, Pittsburgh, PA, 2008, pp. 1-4.

[11] G. A. Taylor, M. R. Irving, P. R. Hobson, C. Huang, P. Kyberd, and R. J. Taylor, "Distributed Monitoring and Control of Future Power Systems via Grid Computing," in Power Engineering Society General Meeting, Montreal, Canada, 2006, pp. 1-5.

[12] S. Ahn and S. Moon, "Economic Scheduling of Distributed Generators in a Microgrid Considering Various Constraints," in IEEE Power \& Energy Society General Meeting, Calgary, Canada, 2009, pp. 1-6.

[13] C. A. Hernandez-Aramburo, T. C. Green, and N. Mugniot, "Fuel Consumption Minimization of a Microgrid," Industry Applications, vol. 41, no. 3, 2005, pp. 673-681.

[14] M. Adamiak, S. Bose, Y. Liu, J. Bahei-Eldin, and J. DeBedout, "Tieline Controls in Microgrid Applications," in Bulk Power System Dynamics and Control - VII. Revitalizing Operational Reliability Symposium, Charleston, SC, 2007, pp. 1-9.

[15] F. Z. Peng, Y. W. Li, and L. M. Tolbert, "Control and Protection of Power Electronics Interfaced Distributed Generation Systems in a Customer-Driven Microgrid," in Power \& Energy Society General Meeting, Calgary, Canada, 2009, pp. 1-8.

[16] Y. Che, Z. Yang, and K. W. Eric Cheng, "Construction, Operation and Control of a Laboratory-Scale Microgrid," in 3rd International Conference Power Electronics Systems and Applications, Hong Kong, 2009, pp. 1-5.

[17] A. L. Dimeas and Nikos D. Hatziargyriou, "Operation of a Multiagen System for Microgrid Control," Power Systems, vol. 20, no. 3, 2005, pp. 1447-1455.

[18] A. Dimeas and N. Hatziargyriou, "A MultiAgent System for Microgrids," Power Engineering Society General Meeting, vol. 1, 2004 pp. $55-58$.

19] "Powermonitor 3000 Product Profile," Jan., 2006. [Online]. Available: http://literature.rockwellautomation.com/idc/groups/literature/documents /pp/1404-pp005_-en-p.pdf. [Accessed: Feb., 2007].
[20] J. C. Warren, "Ethernet/IP Applications for Electrical Industrial Systems," in Industry Applications Society Annual Meeting, Houston, TX, 2009, pp. 1-5.

[21] P. Mahat, Z. Chen, and B. Bak-Jensen, "A Hybrid Islanding Detection Technique Using Average Rate of Voltage Change and Real Power Shift," Power Delivery, vol. 24, no. 2, 2009, pp. 764-771.

[22] H. Gaztanaga, I. Etxeberria-Otadui, S. Bacha, and D. Roye, "Real-Time Analysis of the Control Structure and Management Functions of a Hybrid Microgrid System," IEEE Industrial Electronics 32nd Annual Conference, Paris, France, 2006, pp. 5137-5142.

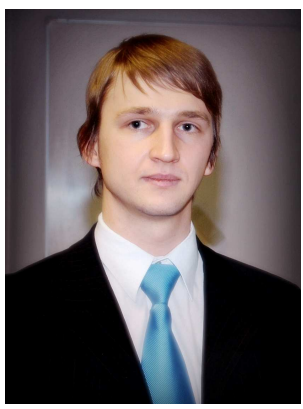

Andriy Palamar received his B.Sc. and M.Sc. degrees in Computer Information Systems and Program Engineering and B.Sc. degree in Management and Business in Production from Ternopil State Technical University, Ternopil, Ukraine, in 2005, 2007 and 2006, respectively. Since 2007 he has been studying for his $\mathrm{PhD}$ at the Ternopil State Technical University. Since 2009 he is a visiting doctoral student at Tallinn University of Technology.

From 2007-2009, he has worked as an Engineer Programmer at the Ltd. "Integral", Ternopil, Ukraine. His research interests include control and monitoring system for microgrids and UPS

e-mail: andrij777@mail.ru

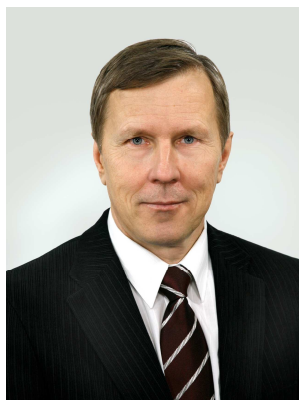

Elmo Pettai received his engineering and Ph.D. degrees in Power Engineering from Tallinn Polytechnic Institute, Tallinn, Estonia, in 1978 and 1985 respectively.

From 1994-2001, he has worked as an expert and managing director in private firms. Currently he is Associate Professor in the Department of Electrical Drives and Power Electronics of Tallinn University of Technology. His research interests include electrical engineering, robotics and industry automation. e-mail:elmo@staff.ttu.ee

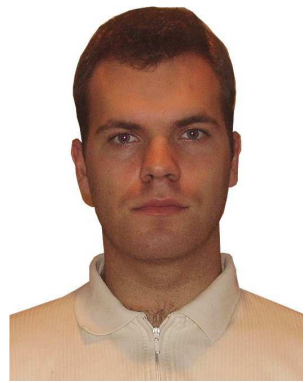

Viktor Beldjajev received his B.Sc. degree in Electrical Drives and Power Electronics in 2007 and M.Sc. degree in the same field in 2009 in Tallinn University of Technology. In 2009 he also received his Dipl. Ing. degree in the field of Automatics in Giessen-Friedberg University of Applied Sciences in Germany. Since 2009 he has been studying for his $\mathrm{PhD}$ at Tallinn University of Technology and also teaching Industrial Robotic.

He has gained diverse experience working in several companies in Germany - EAAT GmbH, LUST Antriebstechnik $\mathrm{GmbH}$, and ABB Flexible Automation $\mathrm{GmbH}$. His research interests include energy quality problems in the industrial field of Smart Grids.

e-mail: vbeldjajev@gmail.com 